\title{
Real-time tumor ablation simulation based on the dynamic mode decomposition method
}

\author{
George C. Bourantas, ${ }^{1}$ Mehdi Ghommem, ${ }^{2}$ George C. Kagadis, ${ }^{3,4, *}$ Konstantinos Katsanos, ${ }^{5}$
}

5 Vassilis C. Loukopoulos, ${ }^{6}$ Vasilis N. Burganos, ${ }^{7}$ and George C. Nikiforidis ${ }^{3}$

${ }^{I}$ MOSAIC Group, Max Planck Institute of Molecular Cell Biology and Genetics, Dresden, Germany

${ }^{2}$ Center for Numerical Porous Media (NumPor), King Abdullah University of Science and Technology (KAUST), Thuwal 23955-6900, Kingdom of Saudi Arabia

$10{ }^{3}$ Department of Medical Physics, School of Medicine, University of Patras, GR 26504 Rion, Greece

${ }^{4}$ Department of Imaging Physics, The University of Texas MD Anderson Cancer Center, Houston, TX 77030, USA

${ }^{5}$ King's College London, Division of Endovascular, Spine \& Interventional Oncology, St Thomas' Hospital, London, United Kingdom

$15{ }^{6}$ Department of Physics, University of Patras, Patras, 26500, Rion, Greece

${ }^{7}$ Institute of Chemical Engineering Sciences-Foundation for Research and Technology, Patras, 26504, Greece

20 *Author to whom correspondence should be addressed:

George C. Kagadis,

Assistant Professor of Medical Physics - Medical Informatics,

Department of Medical Physics,

School of Medicine,

25 University of Patras,

P.O. BOX: 13273, GR 26504, Rion, Greece,

Email: gkagad@gmail.com, George.Kagadis@med.upatras.gr,gkagadis@mdanderson.org.

Tel.: +30 $2610969146,+1312532$ 8834, Fax: +302610969166 
30 Abstract:

Purpose: The Dynamic Mode Decomposition (DMD) method is used to provide a reliable forecasting of tumor ablation treatment simulation in real time, which is quite needed in medical practice. To achieve this, an extended Pennes bioheat model must be employed, taking into account both the water evaporation phenomenon and the tissue damage during tumor ablation.

Methods: A meshless point collocation solver is used for the numerical solution of the governing equations. The results obtained are used by the DMD method for forecasting the numerical solution faster than the meshless solver. The procedure is first validated against analytical and numerical predictions for simple problems. The DMD method is then applied to three-dimensional simulations that involve modeling of tumor ablation and account for metabolic heat generation, blood perfusion, and heat ablation using realistic values for the various parameters.

Results: The present method offers very fast numerical solution to bioheat transfer, which is of clinical significance in medical practice. It also sidesteps the mathematical treatment of boundaries between tumor and healthy tissue, which is usually a tedious procedure with some inevitable degree of approximation. The DMD method provides excellent predictions of the temperature profile in tumors and in the healthy parts of the tissue, for linear and non-linear thermal properties of the tissue.

Conclusions: The low computational cost renders the use of DMD suitable for in-situ real

50 time tumor ablation simulations without sacrificing accuracy. In such a way the tumor ablation treatment planning is feasible using just a personal computer thanks to the simplicity of the numerical procedure that is used. The geometrical data can be provided directly by medical image modalities used in everyday practice

55 Keywords: Bioheat equation, Eulerian, Meshless method, Moving Least Squares, Thermal Ablation. 


\section{Introduction}

60

Image-guided thermal ablation has been gaining popularity as a minimally invasive treatment of localized solid tumors. Energy sources for the delivery of thermal energy to coagulate and destroy cancerous lesions may include radiofrequency ablation (RFA), microwaves (MW), high-intensity focused ultrasound (HIFU) and Light Amplification by

65 Stimulated Emission of Radiation (LASER). Exposure to heat may render cancer cells more sensitive to radiation or even directly attack cancer cells that show reduced sensitivity to radiation. As thermal ablation contributes to the damage of cancer cells, usually with minimal injury to normal tissues, ${ }^{1}$ it is a powerful alternative to more conventional treatment modalities, such as chemotherapy and radiation therapy. ${ }^{2}$ Appropriate medical

70 imaging is the key element for the effective navigation and actuation of ablation instruments and for monitoring and control of treatment outcomes. However, results of thermal ablation therapies depend on the physics of in situ energy deposition and tissueenergy interaction and there is still an unmet need for accurate modeling and prediction of local thermal lesions. Computational bioheat modeling (Computational Heat Transfer; i.e.

75 CHT) prior to thermal ablation of solid tumors holds the promise to project the local ablation isotherms and provide a virtual roadmap for more optimal treatment planning and improved clinical outcomes. In thermal ablation treatment planning simulations, it is important to be able to predict ahead of time the effects of the heating on the tumor and on the surrounding tissue. Theoretical models used for numerical simulations are intended to be used before and during ablation treatments, together with temperature field images obtained by magnetic resonance to predict the effect of source placement and heating. ${ }^{3}$ The majority of bioheat models make use of the Pennes bioheat equation, ${ }^{4,5}$ which is based on the classical Fourier law ${ }^{6}$ and accounts for blood flow through a temperaturedependent heat source term. The Pennes equation works well with acceptable accuracy in

85 the absence of large blood vessels. ${ }^{7}$ A method to account for the heat sink effect that is caused by large blood vessels would be to specify an effective convective heat transfer coefficient along the vessel surface ${ }^{8,9}$ or to include some complex relationship between blood flow dynamics in the vessel and transient temperature. ${ }^{10}$ However, it is widely accepted that despite the development of more complex and rigorous models of heat transfer within a tissue, ${ }^{11}$ the Pennes equation remains a remarkably effective method for modeling heat transfer in tissue during thermal ablation. 
Modeling heat conduction in inhomogeneous media with different thermal properties, like body tissues with pathological regions, is a computationally demanding problem due to its inherent non-linearity. One can assign to it the thermal conductivity of the neighboring media or assume a linear profile of temperature change and satisfy energy conservation in each direction of heat conduction. ${ }^{12}$ The identification of regions with different conductivity and the description of the precise geometry of the boundaries between them are known to necessitate tedious mathematical treatments that complicate the solution of the heat conduction problem. Specifically, the typically used methodology of continuity of heat fluxes at the interface between regions of different conductivity necessitates the calculation of local normal vectors at the tumor/tissue interface, which, in the majority of cases, is a cumbersome procedure. Several theoretical models have appeared in the literature concerning the impact of changes in tissue properties on the resulting temperature profiles, along with the impact of microvascular ${ }^{13}$ and macrovascular perfusion. In fact, microvascular perfusion occurs within the capillary system and affects both the size and the shape of the ablation zone. On the other hand, macrovascular perfusion reflects the heat sink effect of large blood vessels at the site of the ablation zone. Both types of tissue perfusion play a crucial role in the tumor ablation procedure and influence significantly the dimensions of the ablation area. As mentioned above, athough the Pennes bioheat equation

110 does not account for heat loss due to blood flow through large, discrete vessels (heat sink effect), it has been shown to describe the effect of blood perfusion on the temperature distribution with acceptable accuracy. ${ }^{7}$

In the present study, a Dynamic Mode Decomposition (DMD) method in conjunction with the meshless point collocation method has been used in order to numerically solve the

115 transient bioheat transfer equation with temperature dependent properties. The classical Pennes bioheat equation has been extended to incorporate water evaporation and tissue damage during ablation, accounting for temperature-dependent thermal properties of the tissue. This approach treats three-dimensional heat conduction problems within pathological tissues of locally varying conductivity and locally varying blood perfusion rate, viewing them as continuous domains, and solving the corresponding bioheat differential equation with variable coefficients. With the present scheme, the time needed for running a simulation in a standard PC amounts to just a few seconds for high-resolution images. In general, 3D models or MR-aided thermal ablation require remarkable computational resources for the solution of large systems of nonlinear algebraic equations.

125 In particular, for a spatial domain that can be represented by a uniform nodal distribution 
(the arrangement of the pixels in a stack of images) the use of a meshless point collocation method can be very efficient.

In this work the fully non-linear extended Pennes bioheat equation in three dimensions is solved numerically using the DMD method for checking the convergence and the stability of the linearized solver and, finally, for forecasting. The proposed scheme is validated and shown that can be used potentially with both numerical and experimental data. The accuracy is crucial for its later use in real raw data. In this way, the DMD scheme can be directly used in feedback control systems, namely DDDAS (Dynamic Data Driven Application Systems) that integrate computer models with field measurement systems to

135 allow real-time simulation. ${ }^{14}$ The method is validated against a well-established meshless solver of the non-linear Poisson type, extended bioheat equation for applications that incorporate physical phenomena such as water evaporation and tissue damage. The applicability of the method can then be used for medical imaging modalities, such as magnetic resonance thermal imaging (MRTI) that are widely used for tumor ablation

140 monitoring and control. The data will be the output of a thermal MRI and the simulation conducted will be data driven. The numerical scheme is straightforward and easy to implement. It is shown here that the meshless collocation method, which provides both accuracy and convergence for the harmonic operator, produces numerical results for the temperature distribution that are in very good agreement with analytical predictions or with

145 the results of the flux continuity method for different benchmark problems. In addition, it is shown that arbitrary and random conductivity maps can be treated in a straightforward manner with this method, at contrast with the flux continuity technique that is not applicable in the case of vaguely defined boundaries.

The paper is organized as follows. In Section 2, the governing equation of the physical

150 phenomenon under consideration is presented. A brief review of Dynamic Mode Decomposition (DMD) method used is presented in Section 3, along with the algorithmic description of the method. In Section 4, both the computational efficiency and the accuracy of the meshless point collocation method are presented. Further numerical examples that involve heating of three-dimensional tumor geometries within an otherwise healthy tissue

155 are solved in Section 5. Finally, conclusions and discussion complete the paper.

\section{Bioheat equation model}


The most widely used bioheat equation for numerical simulations of thermal therapies is the Pennes bioheat equation, which incorporates the effects of blood perfusion and metabolic heat generation: ${ }^{6}$

165

$$
\rho c \frac{\partial T(\boldsymbol{x}, t)}{\partial t}=\nabla(k(T) \nabla T)+\omega_{b} \rho_{b} c_{b}\left(T_{a}-T\right)+Q_{m}+Q_{r}(\boldsymbol{x}, t)
$$

where $\rho\left[\mathrm{kg} / \mathrm{m}^{3}\right]$ is the mass density, $c[\mathrm{~J} / \mathrm{kg} \mathrm{K}]$ is the specific heat capacity, $k[\mathrm{~W} / \mathrm{K}]$ is the thermal conductivity of the tissue, and $\omega_{b}\left[\mathrm{~kg} / \mathrm{m}^{3} \mathrm{~s}\right], \rho_{b}\left[\mathrm{~kg} / \mathrm{m}^{3}\right]$ and $c_{b}[\mathrm{~J} / \mathrm{kg} \mathrm{K}]$ represent blood perfusion, density, and specific heat of blood, respectively. $T_{a}[K]$ is the arterial temperature, which is here treated as constant, and $T(\boldsymbol{x}, t)$ is the local tissue temperature. $Q_{m}$ $\left[\mathrm{W} / \mathrm{m}^{3}\right]$ is the metabolic heat generation and $Q_{r}(\boldsymbol{x}, t)\left[\mathrm{W} / \mathrm{m}^{3}\right]$ is the spatial heating rate that is provided by an external heat source. From the physical side of view, the first term on the right hand side of the Pennes equation represents heat conduction in the tissue, caused by the temperature gradient and the second term describes the heat transfer between the tissue matrix and local microcirculation. The rest of the terms represent the internal heat generation due to metabolism $\left(Q_{m}\right)$ and the spatial heating caused by external heat sources $\left(Q_{r}\right)$.

\subsection{Extended bioheat equation}

The Pennes bioheat model assumes uniform perfusion rate without accounting for blood flow direction. As a result, it neglects the counter-current arrangement of the circulatory system, taking into consideration only the venous blood stream as the fluid stream equilibrated with the tissue. Additionally, it neglects certain physical phenomena that take place during thermal ablation, namely, water evaporation and thermal tissue damage.

\section{Water evaporation and tissue damage}

At the relatively high temperatures that are reached during ablation, the following phenomena must be taken into account in the bioheat equation: water transport due to temperature changes, changes in local water content due to heating, and water evaporation at high temperatures along with its possible recondensation. ${ }^{15}$ Specifically, water vapor that is generated upon heating the tissue increases the gas pressure locally and, then, escapes to 
lower pressure and lower temperature regions. It also condenses to water liquid and releases its latent heat to the adjacent tissue regions, increasing the temperature locally. Due to the temperature increase, this region gains water content eventually during the condensation process. As experimental observations showed ${ }^{16}$ thermal ablation has several effects on tissue water properties including dependence on temperature and on water content of the tissue. Because of the dearth of valid experimental data, an approach to tackle such a complex problem is to attempt to incrementally improve the model as our understanding of the physical system improves and more information on material properties becomes available.

205 The power density that is used for evaporation is related to the change in water content of the tissue as follows: ${ }^{15}$

$$
Q_{E}=-\beta \frac{\partial W}{\partial t}
$$

210 where $\beta$ is the water latent heat constant, equal to $2260[\mathrm{~kJ} / \mathrm{kg}]$, and $W$ is the tissue water density $\left[\mathrm{kg} / \mathrm{m}^{3}\right]$, which is assumed to be a function of temperature. Using the chain rule, the derivative of $W$ with respect to time is given by

$$
\frac{\partial W}{\partial t}=\frac{d W}{d T} \frac{\partial T}{\partial t}
$$

Substituting this into Eq. (2), yields

$$
Q_{E}=-\beta \frac{d W}{d T} \frac{\partial T}{\partial t}
$$

\section{Tissue damage}

Tissue damage due to heating is a complex function of the amount of temperature elevation and temperature ramp. A crucial matter in this problem is to determine the precise amount of tissue damage during ablation. Several theoretical models have been suggested over the last few years. ${ }^{17}$ However, experimental measurements have shown that blood perfusion in response to elevated temperature is a complex function of both temperature and time ${ }^{18,19}$ Tissue thermal damage due to temperature elevations in the tissue over a threshold value for a period of time can be described by an Arrhenius-type equation: ${ }^{20}$ 
$230 \Omega=\int_{0}^{\tau} P e^{-\frac{\Delta E}{R T(x, \tau)}} d \tau$

where $\Omega$ is a measure of the extent of thermal damage to the tissue, $P$ is a proportionality constant, $\Delta E$ is the activation energy, and $R$ is the universal gas constant. More specifically, the undamaged fraction of the tissue is $f_{u}=\mathrm{e}^{-\Omega}$, whereas the damaged fraction is $f_{d}=1-f_{u}$.

235 Taking into account the above analysis, the extended bioheat equation becomes

$$
\begin{aligned}
&\left(\rho(T) \frac{d c}{d T} T+c(T) \frac{d \rho}{d T} T+\rho(T) c(T)-\beta \frac{d W}{d T}\right) \frac{d T}{d t}=\nabla(k(T) \nabla T)+ \\
& \omega_{b}(T, \Omega) \rho_{b} c_{b}\left(T_{a}-T\right)+Q_{r}+Q_{m}
\end{aligned}
$$

incorporating water evaporation, tissue damage during heating, and temperature dependent thermal properties of the tissue.

\section{Dynamic Mode Decomposition}

\subsection{Historical Background}

In recent decades there have been great advances in the extraction of coherent structures from experiments and numerical simulations. Several techniques, such as balanced truncation, proper orthogonal decompositions (POD) and dynamic mode decomposition (DMD), have been efficiently used for global model reduction, most of which involve projection of the original governing equations onto a set of modes. Proper orthogonal decomposition constitutes a powerful mode decomposition technique for extracting the most energetic structures from a linear or nonlinear dynamical process. ${ }^{21}$ Dynamic mode decomposition (DMD) method has been recently introduced ${ }^{22}$ to accurately extract coherent

255 and dynamically relevant structures. This method enables the computation, from empirical data, of the eigenvalues and eigenvectors of a linear model that best represents the underlying dynamics, even if those dynamics are produced by a nonlinear process. This technique has been successfully applied for the analysis of experimental, ${ }^{23,24}$ and numerical ${ }^{25}$ flow field data and has shown a great capability to capture the relevant associated dynamics. Additionally, one could use the method of snapshots ${ }^{26}$ which allows for a significant reduction of the large data sets. In this method, sets of instantaneous solutions (or snapshots) of the flow parameters obtained from a well-established solver are 
generated and stored in an $M \times N$ matrix, where $M$ and $N$ denote, respectively, the number of grid points and snapshots. The DMD method is based on postprocessing a sequence of snapshots to extract the dynamic information. ${ }^{27,28}$ In such a way, the DMD method can also be used for forecasting. By analyzing and recognizing the dynamical modes of the physical system under investigation, the modes can be used to predict the final output.

\subsection{Mathematical background}

270

To reduce the computational complexity associated with nonlinear dynamical systems, two common mode decomposition methods have been successfully used, namely, dynamic mode decomposition (DMD) and proper orthogonal decomposition (POD). Both of them are based on processing information from a sequence of snapshots (or instantaneous

275 solutions) to identify a low-dimensional set of basis functions. These functions are then used to derive a low-dimensional dynamical system that is typically obtained by Galerkin projection. ${ }^{29-31}$ DMD enables the computation, from simulation and empirical data, of the eigenvalues and eigenvectors of a linear model that best represents the underlying dynamics, even if this dynamics is produced by a nonlinear process. One important feature of this method is its ability to extract dynamic information from flow fields without depending on the availability of a model, but rather is based on a sequence of snapshots. ${ }^{22}$, ${ }^{25}$ The application of this mode decomposition technique is suggested here in order to speed up significantly the numerical simulation of the bioheat equation while predicting the temperature field with good accuracy.

1. Collect and store the snapshots, instantaneous solutions separated by a constant time step $\Delta t$, in two consecutive snapshot sequences as follows:

$$
T_{1}^{n_{s}-1}=\left(\begin{array}{ccc}
\mid & \mid & \mid \\
\boldsymbol{T}_{1} & \cdots & \boldsymbol{T}_{n_{s}-1} \\
\mid & \mid & \mid
\end{array}\right) \text { and } T_{2}^{n_{s}}=\left(\begin{array}{ccc}
\mid & \mid & \mid \\
\boldsymbol{T}_{2} & \cdots & \boldsymbol{T}_{n_{s}} \\
\mid & \mid & \mid
\end{array}\right)
$$

Here, $\boldsymbol{T}_{i} \in \mathbb{R}^{n_{p}}$ is the vector collecting the temperature values at the grid points at time instant $t_{i}=i \Delta t$ where $n_{p}$ denotes the number of grid points and $n_{s}$ is the number of snapshots. In the present study, we assume that $n_{p} \gg n_{s}$. The objective is to evaluate only the first few instantaneous temperature fields to compute the dynamically-relevant structures that enable the forecast of the temperature field with good accuracy while saving significant computational cost. 
2. Perform the singular value decomposition (SVD) of the first snapshot matrix $T_{1}^{n_{s}-1}$; that is, $T_{1}^{n_{s}-1}=\boldsymbol{U} \Sigma \boldsymbol{W}$

3. Evaluate the matrix $\tilde{\boldsymbol{S}}$ as $\tilde{\boldsymbol{S}}=\boldsymbol{U}^{T} T_{2}{ }^{n_{s}} \boldsymbol{W}^{T} \Sigma^{-1}$

4. Compute the eigenvalues and eigenvectors of the matrix $\tilde{S}$; that is, solve the following eigenvalue problem, $\tilde{\boldsymbol{S}} \boldsymbol{X}_{i}=\boldsymbol{D}_{i i} \boldsymbol{X}_{i}$ or $\tilde{\boldsymbol{S}} \boldsymbol{X}=\boldsymbol{X} \boldsymbol{D}$ where $\boldsymbol{D}$ is a diagonal matrix

5. Compute the dynamic mode spectrum $\lambda_{i}=\boldsymbol{D}_{i i}$

6. Compute the unscaled dynamic modes as $\tilde{\boldsymbol{\phi}}=\left\{\tilde{\boldsymbol{\phi}}_{i}\right\}_{i=1}^{r}$ where $\tilde{\phi}_{i}=\boldsymbol{U} \boldsymbol{X}_{i}$ and $r \leq \min \left(n_{p}, n_{s}\right)$

7. Compute the vector $\boldsymbol{d}$ from $\boldsymbol{d}=\left(\tilde{\phi}^{T} \tilde{\phi}\right)^{-1} \tilde{\phi}^{T} \boldsymbol{y}_{1}$

8. Evaluate the scaled dynamic modes as $\phi=\tilde{\phi} \boldsymbol{d}$

9. Approximate the temperature field as $T\left(x, t^{k}\right) \approx \tilde{T}\left(x, t^{k}\right)=\sum_{i=1}^{r} \lambda_{i}^{k} \phi_{i}$

Unlike the POD-based reduced-order model that requires the Galerkin projection of the original governing equations onto an optimal subspace, the DMD-based approximation, as given by Eq. (8), does not require a priori knowledge of the governing equations, but rather only involves the analysis of a set of snapshots.

For the subsequent analysis, to check the capability of the dynamic modes to properly predict the temperature variation, we compute the relative error as the $L_{2}$-norm of the difference between the reference and approximate solutions over the reference one; i.e.,

$\|E\|_{2}=\frac{\|T(x, t)-\tilde{T}(x, t)\|_{2}}{\|T(x, t)\|_{2}}$

\section{Meshless point collocation}

\subsection{Computational Efficiency}

Meshless methods were developed to overcome the drawbacks of the traditional mesh based methods, namely, the meshing itself and the local refinement procedure. Thus, there 
is no predefined interconnectivity between the spatial domain nodes in meshless methods, rendering the local refinement procedure easy and straightforward.

The approximation/interpolation method that is used in the context of meshless methods uses a limited number of nodes for the construction of the shape functions and derivatives of the unknown field functions. These nodes are generally referred to as nearest neighboring nodes, while the process of finding the near nearest nodes is commonly referred to as nearest neighboring nodes searching. These approaches can be categorized into all-pair search, cell-list search, and tree search algorithms. ${ }^{32}$ Herein, we used a cell-list search algorithm mainly due to uniform nodal distribution given by the voxel of the stack of the images. Long-range interactions, where in every node the support domain includes

345 the entire spatial domain, incur a computational cost of $O\left(N^{2}\right)$, rendering their direct computation infeasible for practical simulations. At the expense of computing an approximate solution, this computational cost can be reduced to $O(N \log N)$ or even $O(N)$ using fast $N$-body solvers such as the Barnes-Hut algorithm ${ }^{33}$ or Fast Multipole Methods, ${ }^{34}$ respectively. Additionally, interactions that only involve a local neighborhood can efficiently be computed in $O(N)$ time using fast neighbor lists, such as cell lists ${ }^{35}$ or Verlet lists. ${ }^{36}$ Additionally, thanks to their regular computational structure and fine granularity, meshless methods are suitable for parallelism on streaming multiprocessors, such as Graphics Processing Units (GPU).

\subsection{Meshless code validation}

For validating the meshless numerical solver used, we considered a benchmark example of a rectangular domain subjected to temperature impact. ${ }^{37}$ As it can be seen in Fig. 1, the width of the rectangle along the $x$ direction is $0.03 \mathrm{~m}$ and along the $\mathrm{y}$ direction is $0.08 \mathrm{~m}$.

360 The boundary conditions considered are

$$
\begin{aligned}
& T(x, y, t)=45^{\circ} C, x, y \in(\mathrm{A}) \\
& \frac{\partial T}{\partial \boldsymbol{n}}(x, y, t)=0, x, y \in(B),(D) \\
& T(x, y, t)=37^{\circ} C, x, y \in(C)
\end{aligned}
$$

while the initial condition is $T(x, y, 0)=33^{\circ} C, x, y \in \Omega$. A convergence study took place, obtaining a grid independent numerical solution that was compared with the analytical 
solution. The steady state analytical solution, in the absence of a metabolic heat source is given by [ref]

370

$T(x)=T_{a}+\frac{\left(T_{s}-T_{a}\right) \sinh [\mu(L-x)]+\left(T_{c}-T_{a}\right) \sinh (\mu x)}{\sinh (\mu L)}$

with $\mu=\sqrt{\frac{\omega_{b} \rho_{b} c_{b}}{k}}$.

375 The convergent result is achieved at $t=7500 \mathrm{~s}$ (with time step set to $\delta t=1 \mathrm{~s}$ ). The numerical results of the proposed method are compared against the analytical predictions in Fig. 2, where the temperature profile at $y=0$ is plotted at different time instants. There is an excellent agreement between numerical and analytical predictions for all time instants considered here and for sufficiently long time that, essentially, corresponds to the steady

380 case solution.

As a second example (Fig.3), we considered the bioheat equation in the case where the boundaries between regions of different blood perfusion rates, where is expected to raise numerical difficulties, due to the appearance of the first term on the right hand side of Eq. (1). This is the price for circumventing the definition of boundaries between the two 385 regions, the determination of the normal vector at any position on the boundaries, and the application of the flux continuity condition on the boundaries. The boundary conditions are

$$
\begin{gathered}
q(x, y, t)=0, \quad x, y \text { in } A, C, D \\
T(x, y, t)=37^{\circ} C, \quad x, y \text { in } B
\end{gathered}
$$

390 with

$$
\begin{gathered}
\omega_{b}=\left\{\begin{array}{c}
0.0005 \mathrm{~kg} / \mathrm{s} / \mathrm{m}^{3} \quad x, y \text { outside } R \\
0.002 \mathrm{~kg} / \mathrm{s} / \mathrm{m}^{3} \quad x, y \text { in } R
\end{array}\right. \\
Q_{m}=\left\{\begin{array}{c}
420 \mathrm{~W} / \mathrm{m}^{3} \quad x, y \text { outside } R \\
4200 \mathrm{~W} / \mathrm{m}^{3} \quad x, y \text { in } R
\end{array}\right.
\end{gathered}
$$

for tissue with a tumor, where $R$ is the tumor domain. Figure 4 shows the temperature distribution using the proposed method. 


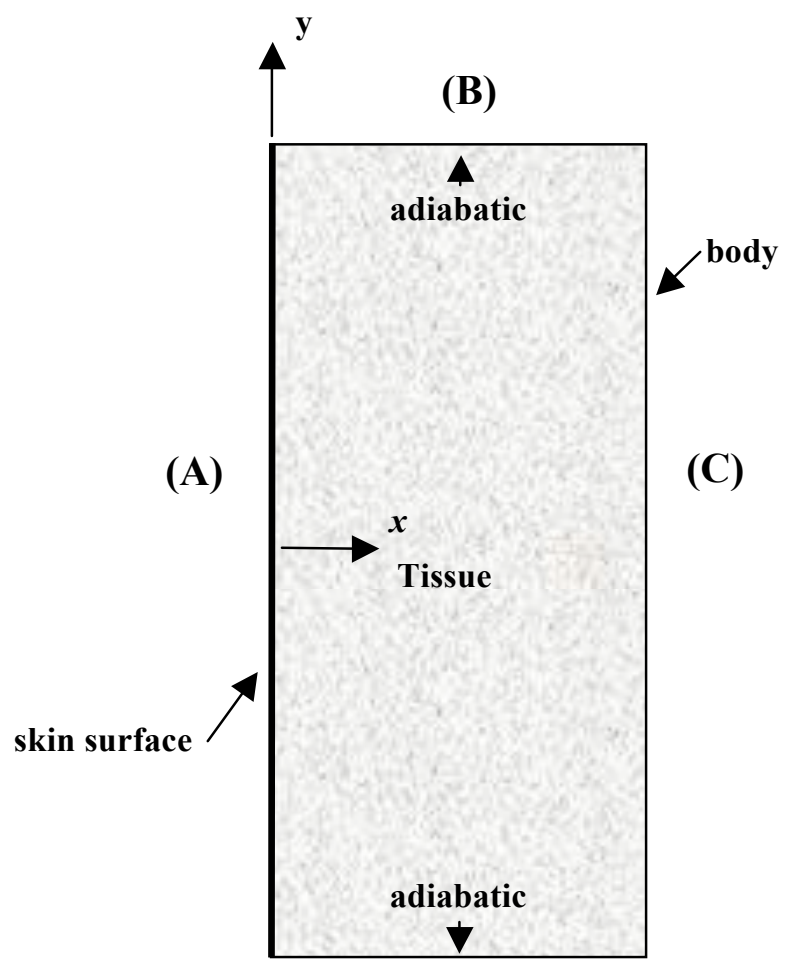

(D)

Figure 1: Geometry of 2D tissue with tumor and boundary conditions for the validation study.

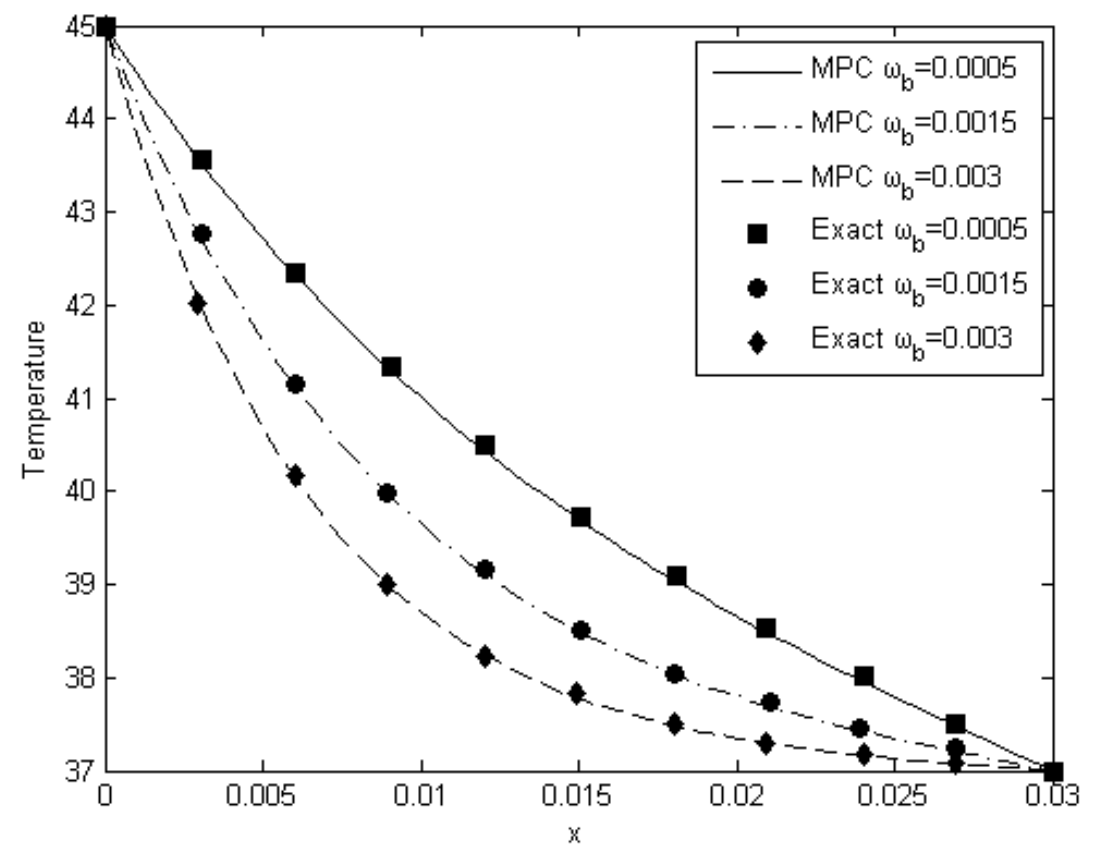


Figure 2: Steady-state temperature distribution along $y=0$ for different blood perfusion rate values.

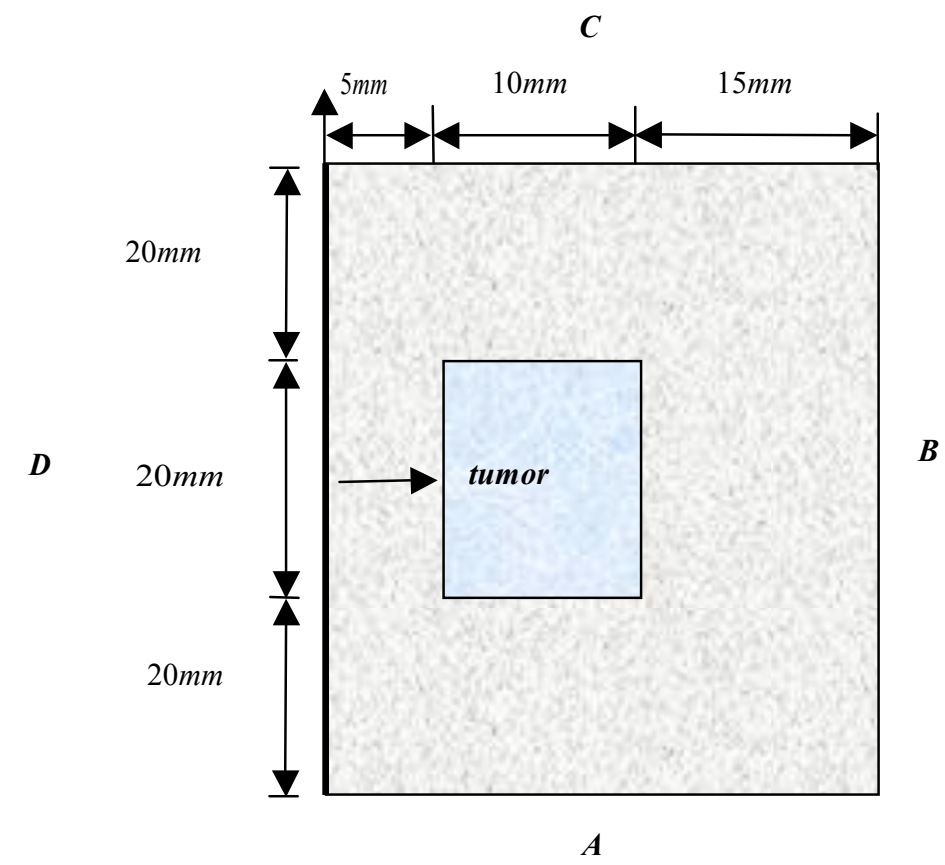

Figure 3: Geometry configuration of the 2D bioheat transfer problem for the validation study.

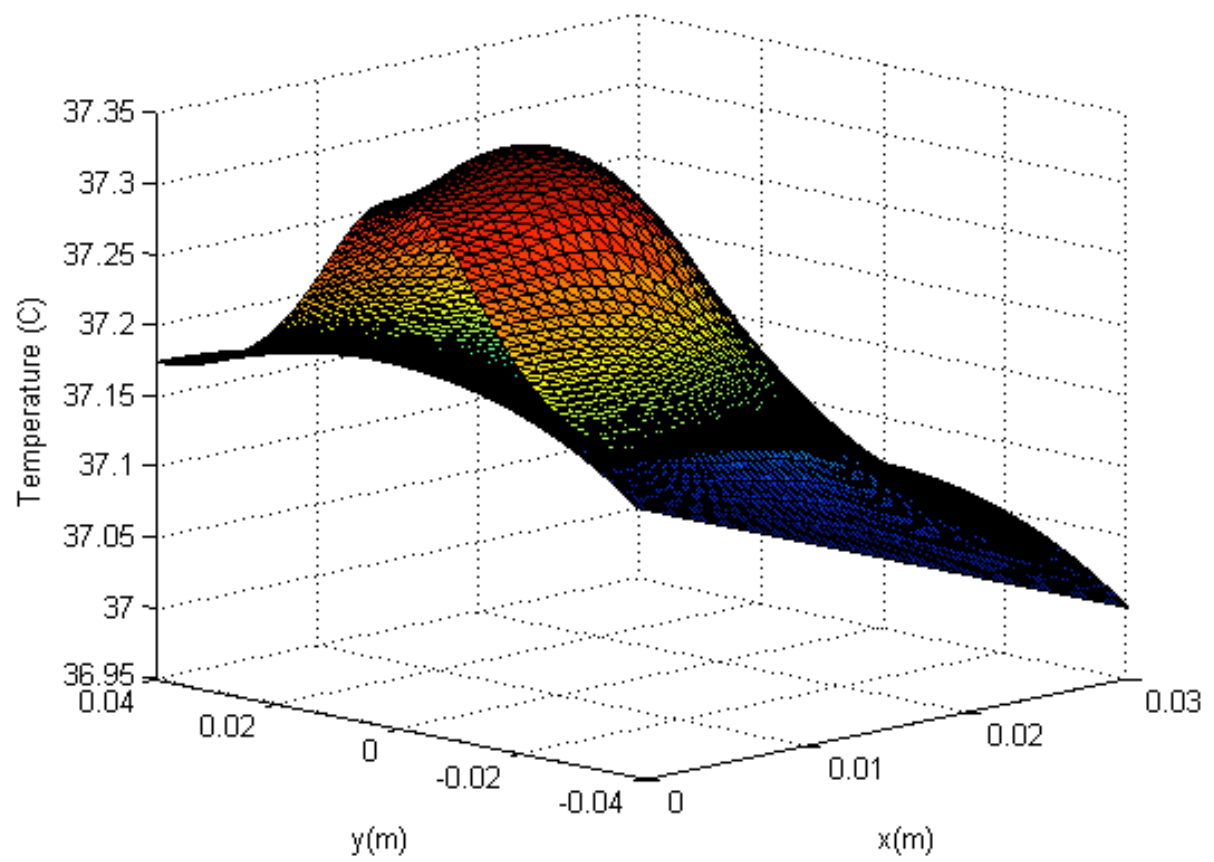


Figure 4: Steady state temperature profile in the tumor region.

Finally, in order to further validate the meshless point collocation scheme, the Pennes equation (Eq. 1) is first solved in the region that is shown in Fig. 5, which is a rectangular region with dimensions $2 L \times L(L=0.05 \mathrm{~m})$ with the tumor located at the center with size $L / 4 \times L / 4$. The physical properties are assigned typical values, namely, thermal conductivity $420 k=0.5 \mathrm{~W} \mathrm{~m}^{-1} \mathrm{~K}^{-1}$, density of both tumor and healthy tissue $\rho=1052 \mathrm{~kg} \mathrm{~m}^{-3}, c_{\mathrm{p}}=3800 \mathrm{~J} \mathrm{~kg}^{-1}$ $\mathrm{K}^{-1}, \eta_{b}=1 \times 10^{-3} \mathrm{~s}^{-1}, Q_{m}=4000 \mathrm{~W} \mathrm{~m}^{-3}$, and a heating source $Q_{r}=100 t \mathrm{~W} \mathrm{~m}^{-3}$ that covers the entire region. At the boundaries, $h=20 \mathrm{~W} \mathrm{~m}^{-2} \mathrm{~K}^{-1}, T_{\mathrm{f}}=20^{\circ} \mathrm{C}$, and $T_{a}=37{ }^{\circ} \mathrm{C}$. The time step is set to $\delta t=20 \mathrm{~s}$, and the initial temperature distribution is obtained from the steady state example, as given in the literature. ${ }^{38}$ Fig. 6 gives the transient temperature profiles, 425 which are compared with the results obtained using the Lattice-Boltzmann (LB) method. ${ }^{38}$ As it can be seen, an excellent agreement between the two methods is obtained. The results of this example show that the MPC method predicts the temperature distribution with very good accuracy and is efficient in dealing with space and time dependent heat sources. As far as the real time needed for the numerical simulations, the results are listed in Table I. 430 For the DMD analysis we took as a snapshot matrix the entire data given by the solver. The forecasting was based on this analysis and so was the time listed. The computational time can be drastically reduced when the snapshot matrix accounts only for a number of steps starting from the initial solution.

Table I: Computational times for MPC and DMD

\begin{tabular}{ccc}
\hline \# of nodes & MPC (sec) & DMD (sec) \\
\hline 7,381 & 53.9 & 4.1 \\
13,041 & 206.3 & 7.1 \\
20,301 & 346.2 & 11.7 \\
29,161 & 427.3 & 19.6 \\
39,621 & 520.8 & 23.7 \\
51,681 & $1,171.1$ & 31.9 \\
\hline
\end{tabular}




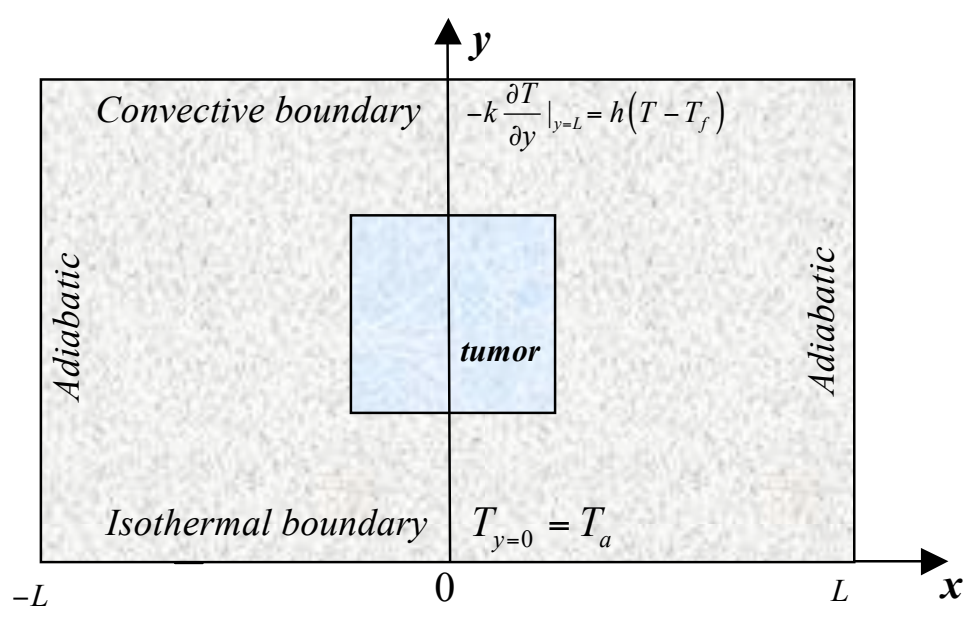

440

Figure 5: Geometry of 2D tissue with tumor and boundary conditions for the third validation study.

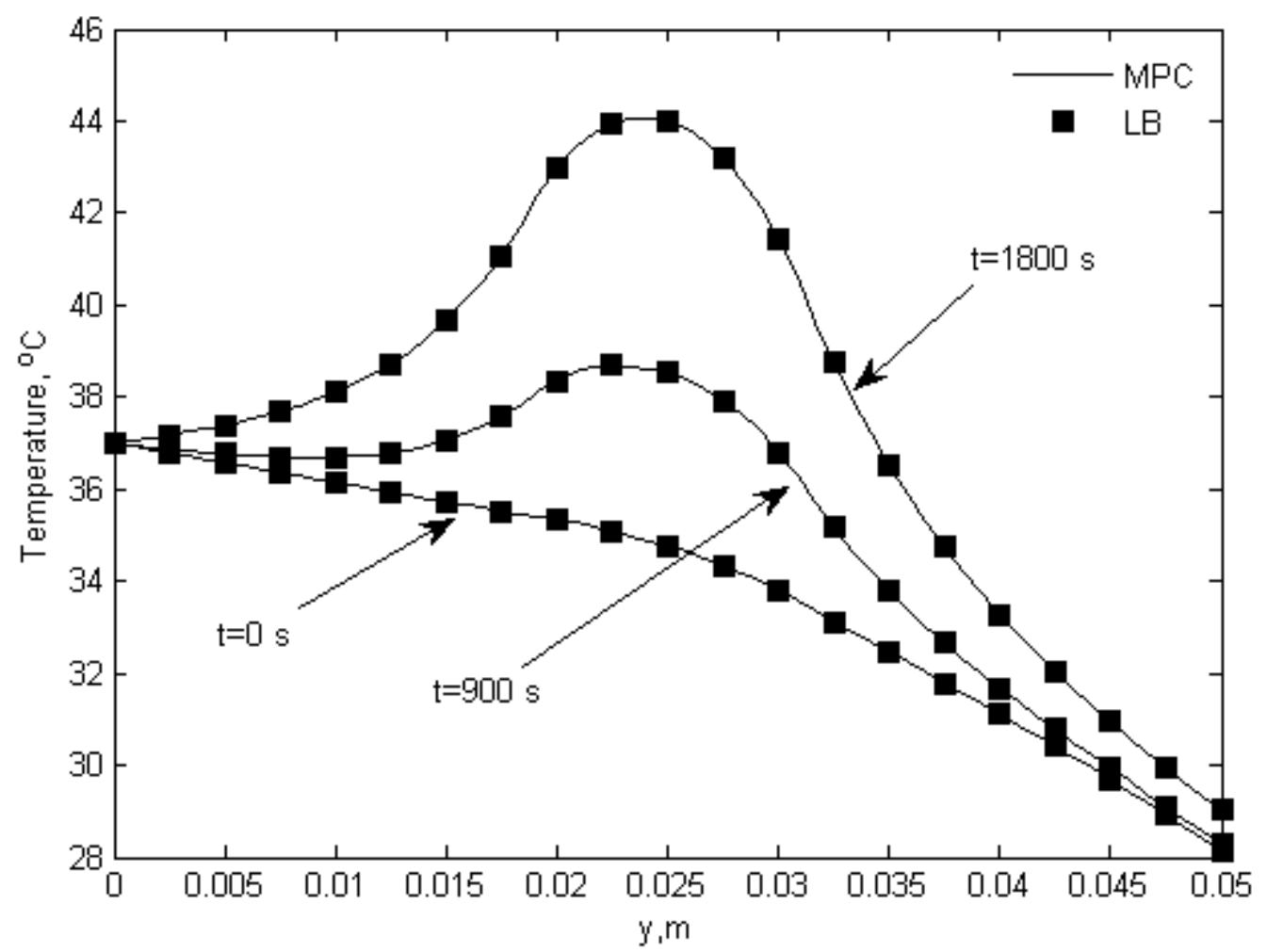

Figure 6: Transient temperature profiles for the case of Fig. 1 along the line $x=0$. Comparison of MPC results (solid lines) with LBM results (symbols, from Zhang, 2008).

\section{Results and Discussion}

Mesh-based methods are widely used in order to solve heat transfer problems in tissues. In that approach subdomains (healthy tissue and tumor) with different thermal conductivities, 
have to be segmented and a different mesh configuration has to be constructed for each of in the majority of the cases is not fully automated and requires the application of the continuity of fluxes along the interfaces of the subdomains. In the present study we adopt an Eulerian type of formulation. In this way there is no need for different mesh configurations between the tumor and the surrounding healthy tissue or adjacent organs.

460 We simulated rectangular geometries with the tumor given also as a rectangular domain, assigning thermal conductivity values directly to grid points. As a result, the proposed scheme can be easily applied to cases where the grid is dictated directly by the raw data provided a mapping between the intensity values and the thermal conductivities is available. In more details the data sites needed for the numerical solution of the governing

465 equations using meshless methods are directly extracted from image data. The meshless method is based only on a set of nodes with no predefined inter-connectivity. These are exactly the voxels defined by the raw data. In mesh-based methods, a mesh has to be constructed and the different regions defined have to be segmented. Additionally, the appropriate selection of modes is an important step in extracting the relevant dynamics 470 when applying both the DMD for model reduction.

\section{1 $2 D$ and $3 D$ linear case}

The case of a two-dimensional tumor ablation test is considered next, using the geometry of Fig. 1 and the boundary conditions described in the code validation section, namely, fixed temperature at the lower border, convection at the upper border, and adiabatic side surfaces. A scenario that simulates a real life medical practice is investigated. Specifically, tumor 480 ablation takes place using a heat source of finite volume located in the middle of the tumor, with a radius of $0.002 \mathrm{~m}$ and $Q_{r}=800 \mathrm{t} \mathrm{Wm}^{-3}$. A single heat source is located at the middle $(0.0,0.025 \mathrm{~m})$ of the spatial domain with radius of $0.0025 \mathrm{~m}$, whereas considering a more realistic medical scenario two more heating sources $\left(Q_{r}=800 \mathrm{t} \mathrm{Wm}^{-3}\right)$ were added during the simulation study, located near two corners of the tumor, the first one at $(-0.0055 \mathrm{~m}, 0.022$ $485 \mathrm{~m})$ and the second one at $(0.0055 \mathrm{~m}, 0.022 \mathrm{~m})$ having a common radius of $0.0025 \mathrm{~m}$. The sources were activated with a time lag of $400 \mathrm{~s}$ and $900 \mathrm{~s}$ from the central source, respectively. As a more realistic case, we considered the temperature dependence of all major physical and thermal properties of the tissue. In this case, the bioheat problem 
becomes non-linear and the numerical solution of the bioheat equation becomes more thermal properties have been reported, the thermal properties of the tissue can be expressed in the following way: ${ }^{39}$

$$
\begin{gathered}
c(T)=4.19\left(0.37+0.63 \lambda_{c} w\right) * 10^{3}(\mathrm{~J} / \mathrm{kg} \mathrm{K}) \\
k(T)=4.19\left(0.133+1.36 \lambda_{k} w\right) * 10^{-1}(\mathrm{~W} / \mathrm{m} \mathrm{K}) \\
\rho(T)=\left(1.3+0.3 \lambda_{\rho} w\right) * 10^{3}\left(\mathrm{~kg} \mathrm{~m}^{-3}\right)
\end{gathered}
$$

where $w$ is the percentage of the mass of water in the tissue, approximately $69 \%$ for liver tissue, $T\left({ }^{\circ} \mathrm{C}\right)$ is the tissue temperature, and $\lambda_{c}, \lambda_{k}, \lambda_{\rho}$ are temperature dependent factors for the specific heat, thermal conductivity, and density of water in the range of $20-100{ }^{\circ} \mathrm{C}:{ }^{39}$

$$
\begin{aligned}
& \lambda_{c}=1.016 * 10^{-4}\left(T-20^{\circ} \mathrm{C}\right) \\
& \lambda_{k}=1+1.78 * 10^{-3}\left(T-20^{\circ} \mathrm{C}\right) \\
& \lambda_{\rho}=1-4.98 * 10^{-4}\left(T-20^{\circ} \mathrm{C}\right)
\end{aligned}
$$

Initially, the temperature was set to $T_{\text {init }}=37^{\circ} \mathrm{C}$ for the entire spatial domain, while the boundary conditions remain the same as in the previous case. A lagging of coefficients procedure is used in order to linearize the non-linear bioheat equation. The total simulation time is set equal to $30 \mathrm{~min}$ and the time step $\delta t$ is set equal to $1 \mathrm{sec}$, ensuring the stability of the numerical method considered in the present study. We note that the same time step has been used for both meshless point collocation and DMD-based methods. The above problem is also integrated numerically using the meshless point collocation method. As for the reduced-order model obtained following the DMD-based approach, we use the first four modes computed from the first 40 snapshots as detailed in Section 3.2. We note that increasing the number of snapshots would decrease even more the error but the aim of this study is to show the potentiality of the DMD technique to detect the dominant modes that govern long-term dynamics from a small set of snapshots and to forecast the evolution of the temperature field with an acceptable accuracy. Furthermore, the appropriate selection of 515 modes is an important step in extracting the relevant dynamics when applying both the DMD for model reduction. Increasing the number of modes is expected to improve the accuracy of the DMD-based approximation however this is at the price of increasing the corresponding computational cost. As such, in the present study, we picked only few 
modes so that the reduced-order model is quite fast while maintaining a good predictive 520 capability of the temperature field.

The contour plots of the temperature at different times are shown in Fig. 7. The numerical results obtained from the two methods, DMD and meshless point collocation, were compared to each other, revealing a good agreement. In fact, for the non-linear case, the relative error $\|\mathrm{E}\|_{2}$ is equal to $\mathbf{1 . 0 8} \mathbf{1 0}^{-3}$. The total $\mathrm{CPU}$ time taken to obtain the approximate 525 temperature field shown in Figure 4(b) is $\mathbf{1 . 1 3} \mathbf{~ s}$; that is, $\sim \mathbf{5} \%$ of the total time taken to obtain the fully-resolved solution. These observations show the capability of DMD modes to capture the main characteristics of the temperature evolution and forecast the temperature field with good accuracy for long time periods while reducing significantly the associated computational cost.

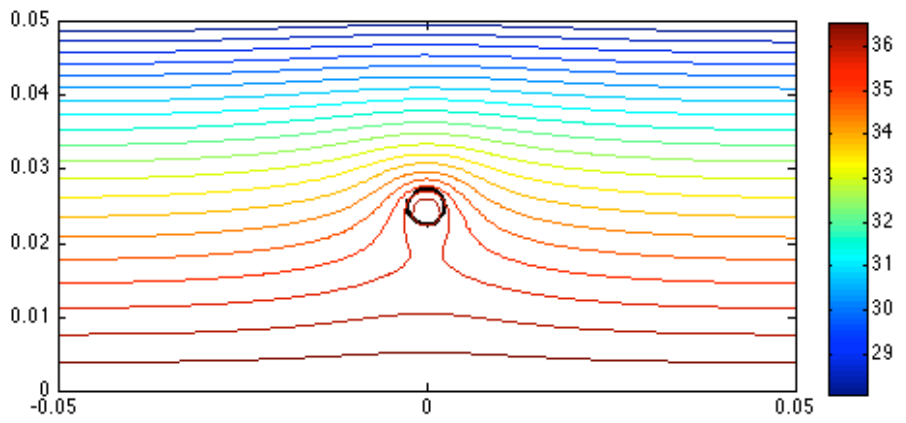

(a)

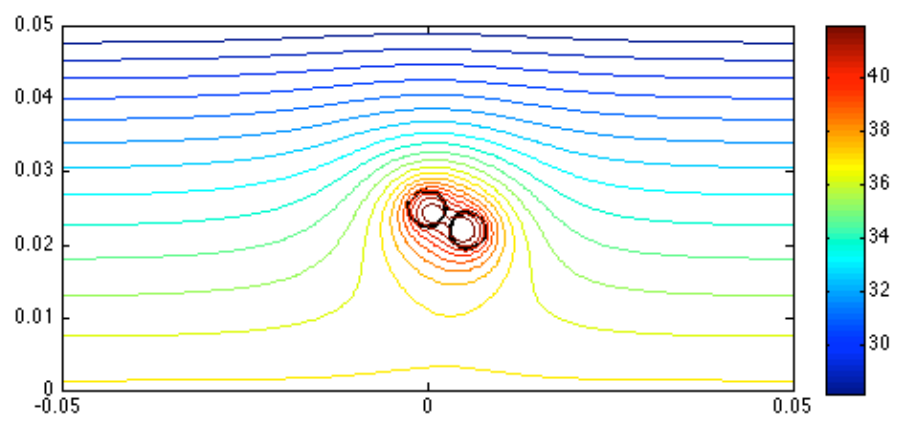

(b)

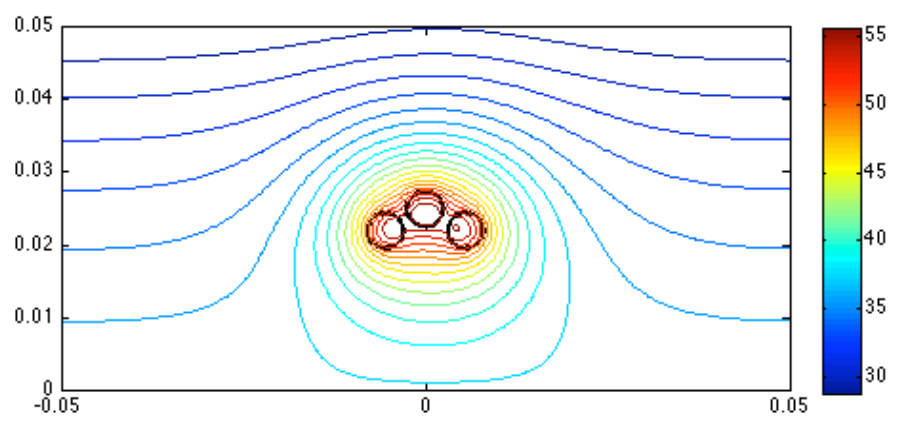


(c)

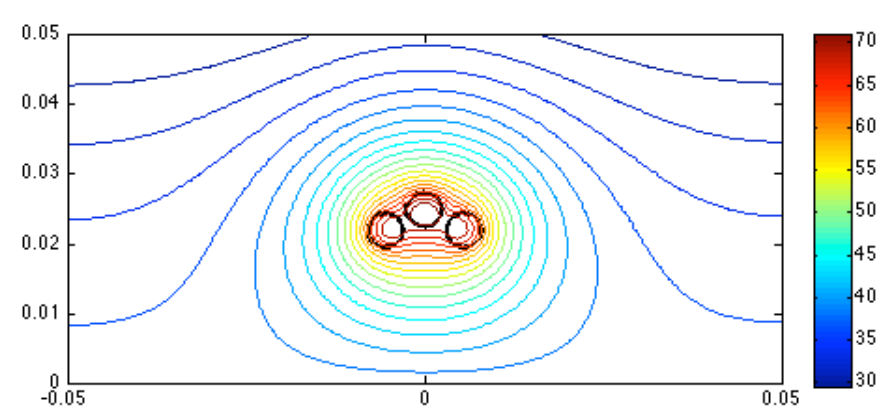

(d)

530

Figure 7: Temperature contour plots at (a) 200s (b) 600s (c) 1200s and (d) 1800s for the case of multiple heat sources and temperature-dependent thermal properties obtained by the DMD-based approximation.

535 Next, we consider the 3D case of the aforementioned heat problem that is, evidently, more computationally-demanding. Again, the goal is to speed up the numerical simulations while keeping a satisfactory level of accuracy. Following a similar approach as shown above, we compute the first 40 modes and use these modes to approximate the temperature field, as given by Eq. 7. A larger number of modes (in comparison with the 2D case) was required

540 to capture the relevant dynamics and achieve good approximation. In Figure 8, we plot the numerical solution at different time steps, as this is reproduced using the DMD method, based on (snapshot matrix) the solution obtained using the meshless point collocation method. The two solutions compare very well. The relative error $\|E\|_{2}$ is equal to $\mathbf{4 . 8} \mathbf{1 0}^{-4}$. As for the reduction in the computational cost, the total CPU time taken to obtain the 545 approximate temperature field shown in Fig. 5(b) is $\mathbf{1 . 3 7}$ s; that is, $\sim \mathbf{0 . 7 6} \%$ of the total time taken to obtain the fully-resolved solution shown in Fig. 5. Clearly, the ability of DMD to extract the relevant dynamic information through analyzing the first few instantaneous solutions makes it an efficient model reduction tool that enables significant savings of computational cost. This level of execution times allows the realization of real-

550 time tumor ablation simulations and the performance of fast and reasonably accurate sweeping over different configurations, thus paving the way to improved treatment planning. 


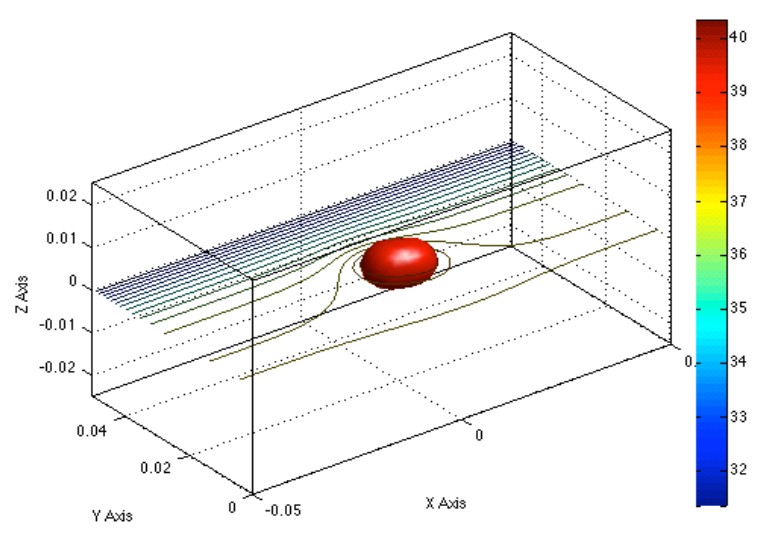

(a)

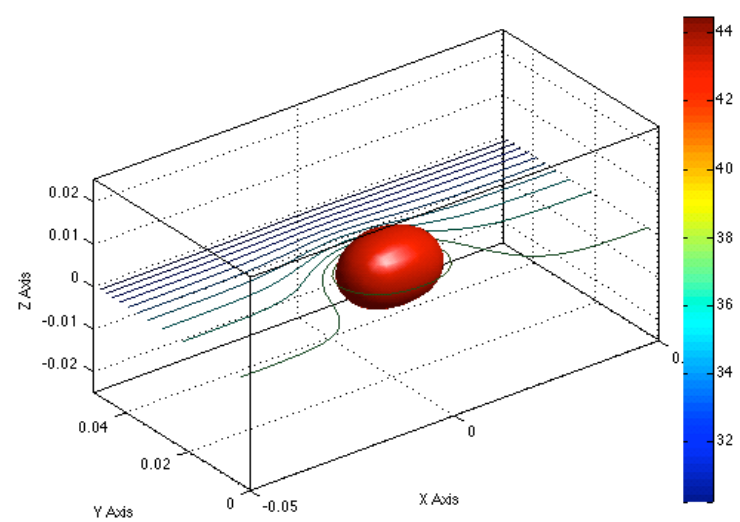

(b)

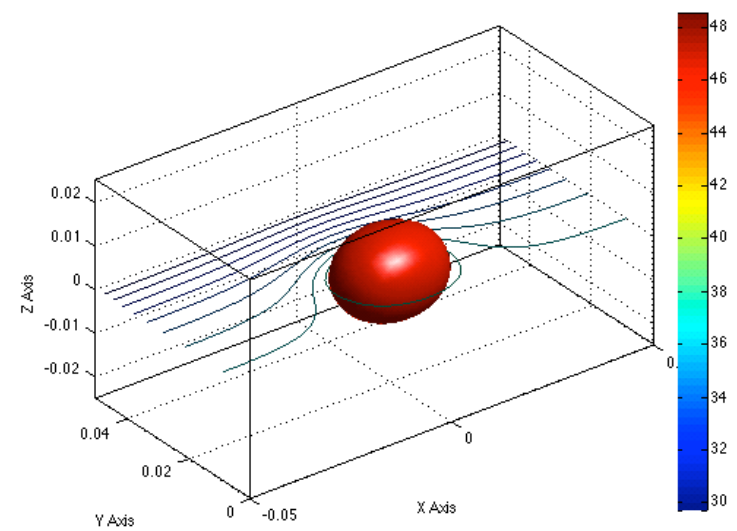

(c)

Figure 8: Temperature iso-contour value $\left(\mathrm{T}=38^{\circ} \mathrm{C}\right)$ plots at (a) 600s (b) $1200 \mathrm{~s}$ and (c

5551800 s for the case of multiple heat sources and temperature-dependent thermal properties obtained by the DMD-based approximation. 


\section{2. $3 D$ non-linear case}

560 Utilizing the DMD method we managed to solve the extended bioheat equation in 3D, taking into account water evaporation, tissue damage, and temperature dependence of all major physical and thermal properties of the tissue and the tumor. In this case, the bioheat problem becomes non-linear and the numerical solution becomes more complicated. The governing equation can be simplified to:

565

$$
\left(\rho(T) c(T)-\beta \frac{d w}{d T}\right) \frac{d T}{d t}=\nabla(k(T) \nabla T)+\omega_{b}(T, \Omega) \rho_{b} c_{b}\left(T_{a}-T\right)+Q_{r}+Q_{m}
$$

Note that the thermal properties of both the tissue and the tumor are functions of temperature $(T)$ and the water content $(w)$. Using an empirical function for the dependence of the tissue water content on temperature to fit experimental data, ${ }^{15}$ one gets

$$
w(T)=\left\{\begin{array}{cc}
0.728+8.487 e^{-2\left(\frac{\mathrm{T}-123.286}{16.634}\right)^{2}}, & \mathrm{~T} \leq 103 \\
0.024+\frac{4.531}{1+e^{\left(\frac{\mathrm{T}-30.173}{28.012}\right)^{2}}}, & \mathrm{~T}>103
\end{array}\right.
$$

Dynamic changes in blood perfusion rate with temperature and damage can be formulated 575 as

$$
\omega_{b}(T, \Omega)=\omega_{b 0} f_{T} f_{u}
$$

where $\omega_{\mathrm{b} 0}$ is the constitutive perfusion rate and $f_{u}=1-f_{T}$, with $f_{T}$ a dimensionless function

580 that accounts for vessel dilation at slightly elevated temperatures, which can be approximated as: ${ }^{40}$

$$
f_{T}=\left\{\begin{array}{cc}
4+0.6\left(\mathrm{~T}-42^{\circ} \mathrm{C}\right) & 37^{\circ} \mathrm{C} \leq T<42^{\circ} \mathrm{C} \\
4 & T \geq 42^{\circ} \mathrm{C}
\end{array}\right.
$$

585 The meshless point collocation method is used in order to simulate tumor ablation and, providing data for the snapshot matrix used in the DMD method. The extended bioheat equation is used to model the thermal distribution in the kidney, using a cubic geometry of 
length $0.02 m$ ranging from $-0.01 m \leq \mathrm{x}, \mathrm{y}, \mathrm{z} \leq 0.01 \mathrm{~m}$ and assuming adiabatic boundaries at all six faces. A scenario of tumor ablation takes place using a heat source of finite volume located at the middle of the tumor with tumor radius $0.002 \mathrm{~m}$. The symmetric geometry setup has been used in order to better impose the thermal boundary conditions. For the latter the physical choices are two; the first is the Dirichlet boundary conditions, where the temperature far away from the source has a constant temperature, that of the surrounding tissues (in most of the cases the temperature chosen is $\mathrm{T}=37^{\circ} \mathrm{C}$ ), and the second one is zero fluxes (meaning zero temperature gradients) at the boundaries, ensuring a dynamic equilibrium. In both cases, the length of the surrounding box has to be large enough, compared with the dimensions of the tumor and, also, quite far from the source. The physical choice for this is to use a square box (symmetrical one) instead of a rectangular one. This won't affect the DMD method, since it relies only on the number of nodes used to 600 define the snapshot matrix. In fact, the latter is only affected on the physical problem itself and not on the numerical method used. The computation time is based on the number of nodes and the convergence on the eigenvalues, meaning the physical problem and the governing equations of the physical phenomenon in consideration. The heat source has a direct action area of radius $0.00025 \mathrm{~m}$ and operates for a time period of $1800 \mathrm{~s}$ with $Q_{r}=750 / 8 \mathrm{MWm}^{-3}$. Initially, the temperature was set to $T_{0}=37^{\circ} \mathrm{C}$ for the entire spatial domain, while the boundary conditions remain the same as in the previous case. A total time was set at $1800 \mathrm{~s}$. The test case was solved using the DMD method using the first few instantaneous solutions to compute the first four modes (i.e., $r=40$ ) as shown above. The numerical results obtained using the meshless point collocation were compared with those

610 obtained from the DMD-based approximation. Fig. 9 shows the iso-contour value of $\mathrm{T}=65^{\circ} \mathrm{C}$, obtained using the DMD method, at different time steps of the ablation treatment, For the DMD calculations only four modes were used. The relative error $\|\mathrm{E}\|_{2}$ is equal to $\mathbf{2 . 2 3} \mathbf{1 0}^{-3}$ and the total CPU time is $\mathbf{2 . 3 4} \mathrm{s}$; that is, $\sim \mathbf{5} \%$ of the total time taken to obtain the fully-resolved solution. In this fully non-linear case the DMD modes managed to 615 capture the main characteristics of the temperature evolution and to forecast the temperature field with good accuracy for long time periods while reducing significantly the associated computational cost. 


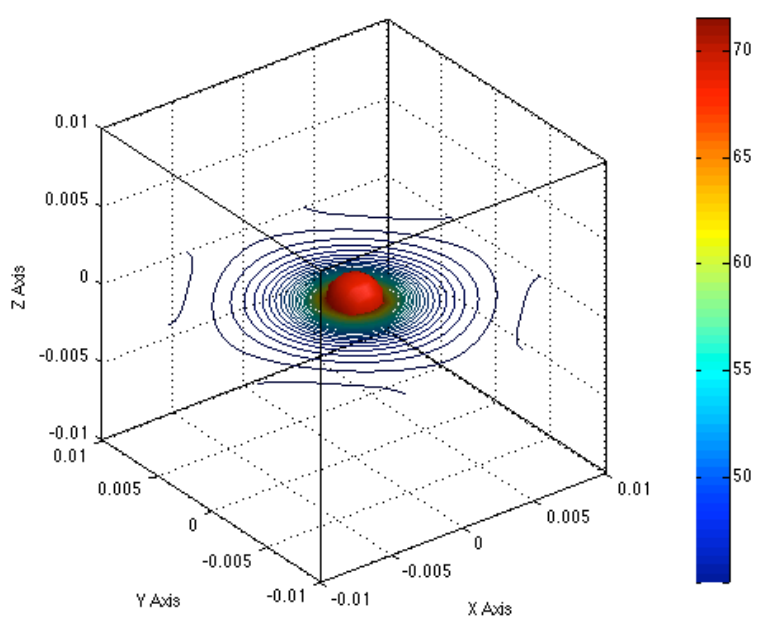

(a)

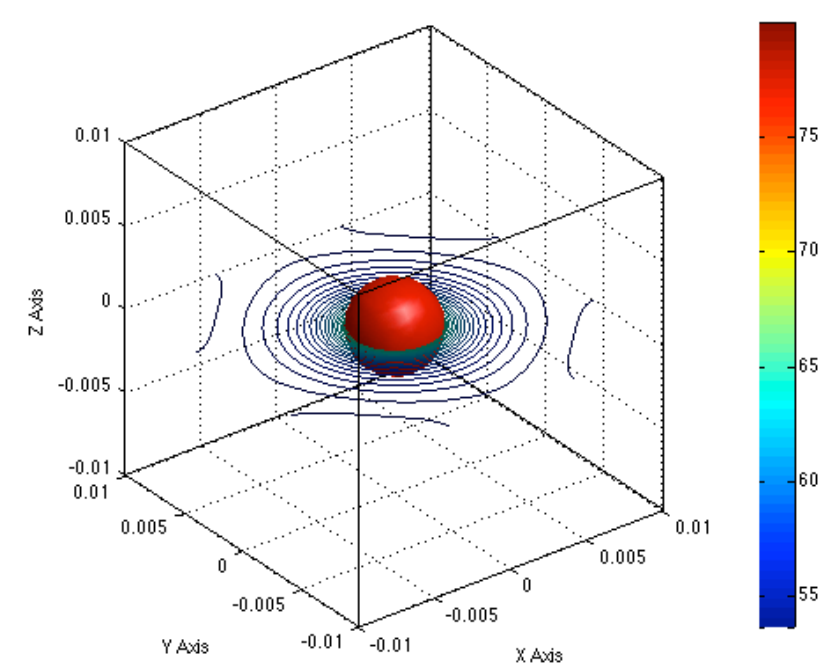

(b)

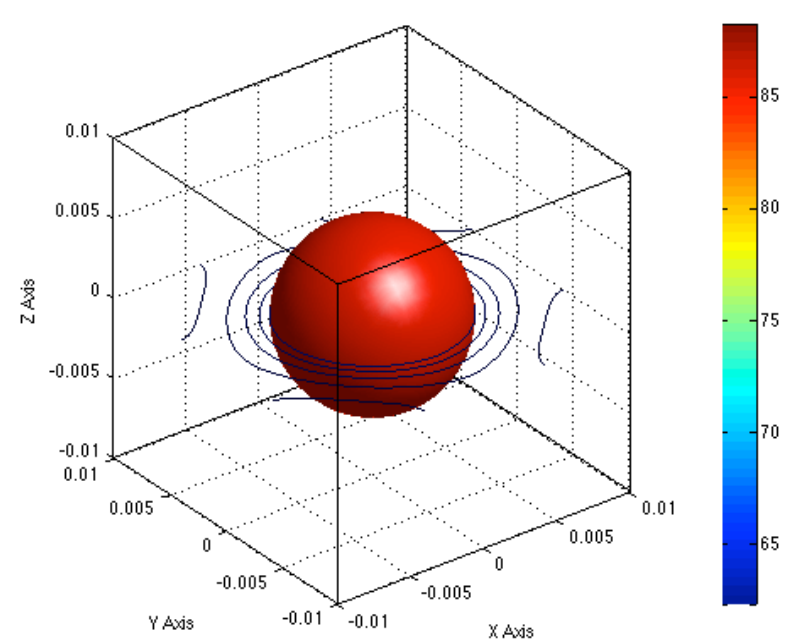

(c) 
625 Figure 9: Temperature iso-contour value $\left(\mathrm{T}=38^{\circ} \mathrm{C}\right)$ plots at (a) $600 \mathrm{~s}$ (b) $1200 \mathrm{~s}$ and (c) 1800 s using the DMD-based approximation.

\section{Conclusions}

630 A Dynamic Mode Decomposition algorithm was employed to solve transient bioheat transfer problems using an Eulerian-type approximation. Both the classical and the extended bioheat equation were solved numerically for several representative cases using the thermal conductivity distribution throughout the spatial domain. To illustrate the applicability and the efficiency of the proposed method in medical practice, several typical

635 examples that appear in therapeutic treatments are considered, which include ablation of a tumor that is surrounded by the healthy part of the tissue, in two and three dimensions. It was found that the method provides excellent predictions of the temperature profile in tumors and in the healthy parts of the tissue, for linear and non-linear thermal properties of the tissue. The very low computational demands and, in particular the very short

640 computational time, renders the DMD method suitable for in-situ real time tumor ablation simulations. In such a way the tumor ablation treatment planning is feasible using just a personal computer thanks to the simplicity of the numerical procedure used. The geometrical data can be provided directly by medical image modalities used in everyday practice, such as CT, MRI, US. Semi-empirical formulas of the tissue thermal properties

645 were used in the present simulations. The results show that the proposed method is an efficient numerical method, easy to follow, with a simple solution procedure, with high accuracy and stability, capable of predicting the steady state and the transient thermal behavior of inhomogeneous materials at greatly reduced computational time. 


\section{Captions to figures}

660 Figure 1: Geometry configuration of the 2D bioheat transfer problem for the validation study.

Figure 2: Steady-state temperature distribution along $y=0$ for different blood perfusion rate values.

665

Figure 3: Geometry of 2D tissue with tumor and boundary conditions for the validation study.

Figure 4: Steady state temperature profile in the tumor region.

670

Figure 5: Geometry of 2D tissue with tumor and boundary conditions for the third validation study.

Figure 6: Transient temperature profiles for the case of Fig. 1 along the line $x=0$.

675 Comparison of MPC results (solid lines) with LBM results (symbols, from Zhang, 2008).

Figure 7: Temperature contour plots at (a) 200s (b) 600s (c) 1200s and (d) 1800s for the case of multiple heat sources and temperature-dependent thermal properties obtained by the DMD-based approximation.

680

Figure 8: Temperature iso-contour value $\left(\mathrm{T}=38^{\circ} \mathrm{C}\right)$ plots at (a) $600 \mathrm{~s}$ (b) $1200 \mathrm{~s}$ and (c 1800 s for the case of multiple heat sources and temperature-dependent thermal properties obtained by the DMD-based approximation.

685

Figure 9: Temperature iso-contour value $\left(\mathrm{T}=38^{\circ} \mathrm{C}\right)$ plots at (a) $600 \mathrm{~s}$ (b) $1200 \mathrm{~s}$ and (c) 1800 s using the DMD-based approximation.

690 


\section{References}

1. J. van der Zee, "Heating the patient: a promising approach?," Annals of oncology : official journal of the European Society for Medical Oncology / ESMO 13, 11731184 (2002).

2. H. R. Moyer and K. A. Delman, "The role of hyperthermia in optimizing tumor 705 response to regional therapy," International journal of hyperthermia : the official journal of European Society for Hyperthermic Oncology, North American Hyperthermia Group 24, 251-261 (2008).

3. W. Wulff, "The endrgy conservation equation for living tissue," IEEE transactions on bio-medical engineering, 494-495 (1974).

710 4. H. W. Huang, "Influence of blood vessel on the thermal lesion formation during radiofrequency ablation for liver tumors," Medical physics 40, 073303 (2013).

5. D. J. Schutt and D. Haemmerich, "Effects of variation in perfusion rates and of perfusion models in computational models of radio frequency tumor ablation," Medical physics 35, 3462-3470 (2008).

715 6. H. H. Pennes, "Analysis of tissue and arterial blood temperatures in the resting human forearm," Journal of applied physiology 1, 93-122 (1948).

7. H. Arkin, L. X. Xu and K. R. Holmes, "Recent developments in modeling heat transfer in blood perfused tissues," IEEE transactions on bio-medical engineering 41, 97-107 (1994).

720 8. I. dos Santos, D. Haemmerich, S. Pinheiro Cda and A. F. da Rocha, "Effect of variable heat transfer coefficient on tissue temperature next to a large vessel during radiofrequency tumor ablation," Biomedical engineering online 7, 21 (2008).

9. Y. J. Liu, A. K. Qiao, Q. Nan and X. Y. Yang, "Thermal characteristics of microwave ablation in the vicinity of an arterial bifurcation," International journal of hyperthermia : the official journal of European Society for Hyperthermic Oncology, North American Hyperthermia Group 22, 491-506 (2006).

10. J. Pearce, "relationship between Arrhenius models of thermal damage and the CEM 43 thermal dose," Proceedings of SPIE, (2009).

11. P. Prakash, "Theoretical modeling for hepatic microwave ablation," The open

12. T. Samaras, A. Christ and N. Kuster, "Effects of geometry discretization aspects on the numerical solution of the bioheat transfer equation with the FDTD technique," Physics in medicine and biology 51, N221-229 (2006).

13. S. L. Brown, J. W. Hunt and R. P. Hill, "Differential thermal sensitivity of tumour and normal tissue microvascular response during hyperthermia," International journal of hyperthermia : the official journal of European Society for Hyperthermic Oncology, North American Hyperthermia Group 8, 501-514 (1992).

14. D. Fuentes, J. T. Oden, K. R. Diller, J. D. Hazle, A. Elliott, A. Shetty, et al., "Computational modeling and real-time control of patient-specific laser treatment of

15. D. Yang, M. C. Converse, D. M. Mahvi and J. G. Webster, "Measurement and analysis of tissue temperature during microwave liver ablation," IEEE transactions on bio-medical engineering 54, 150-155 (2007).

16. P. R. Stauffer, F. Rossetto, M. Prakash, D. G. Neuman and T. Lee, "Phantom and animal tissues for modelling the electrical properties of human liver," International journal of hyperthermia : the official journal of European Society for Hyperthermic Oncology, North American Hyperthermia Group 19, 89-101 (2003). 
17. W. C. Dewey and C. J. Diederich, "Hyperthermia classic commentary: 'Arrhenius relationships from the molecule and cell to the clinic'," International journal of hyperthermia : the official journal of European Society for Hyperthermic Oncology, North American Hyperthermia Group 10, 457-483 (2009).

18. X. He, S. McGee, J. E. Coad, F. Schmidlin, P. A. Iaizzo, D. J. Swanlund, et al., "Investigation of the thermal and tissue injury behaviour in microwave thermal therapy using a porcine kidney model," International journal of hyperthermia : the official journal of European Society for Hyperthermic Oncology, North American Hyperthermia Group 20, 567-593 (2004).

19. M. van Vulpen, B. W. Raaymakers, A. A. de Leeuw, J. B. van de Kamer, R. J. van Moorselaar, M. G. Hobbelink, et al., "Prostate perfusion in patients with locally advanced prostate carcinoma treated with different hyperthermia techniques," The Journal of urology 168, 1597-1602 (2002).

20. A. J. Welch and M. J. C. Van Gemert, Optical-thermal response of laser-irradiated tissue, edited. (Plenum Press, New York, NY, 1995).

21. Z. Wang, I. Akhtar, J. Borggaard and T. Iliescu, "Two-level discretizations of nonlinear closure models for proper orthogonal decomposition," Journal of

22. P. J. Schmid, "Dynamic mode decomposition of numerical and experimental data," Journal of Fluid Mechanics 656, 5-28 (2010).

23. D. Duke, J. Soria and D. Honnery, "An error analysis of the dynamic mode decomposition," Experiments in Fluids, 529-542 (2012).

770 24. P. J. Schmid, "Application of the dynamic mode decomposition to experimental data," Experiments in Fluids, 1123-1130 (2011).

25. P. J. Schmid, L. X. Li, M. P. Juniper and O. Pust, "Applications of the dynamic mode decomposition," Theoretical and Computational Fluid Dynamics 25, 249-259 (2011).

775 26. A. L. Sirovich, "Turbulence and the dynamics of coherent structures," Quarterly of Applied Mathematics 45, 561-590 (1987).

27. M. Ghommem, V. M. Calo and Y. Efendiev, "Mode decomposition methods for flows in high-contrast porous media. A global approach," Journal of Computational Physics 257, 400-413 (2014).

780 28. M. Ghommem, M. Presho, V. M. Calo and Y. Efendiev, "Mode decomposition methods for flows in high-contrast media. A global-local approach," Journal of computational physics 253, 226-238 (2013).

29. I. Akhtar, A. H. Nayfeh and C. J. Ribbens, "On the stability and extension of reduced-order Galerkin models in incompressible flows: A numerical study of vortex shedding," Theoretical and Computational Fluid Dynamics 23, 213-237 (2009).

30. I. Akhtar, Z. Wang, J. Borggaard and T. Iliescu, "A new closure strategy for proper orthogonal decomposition reduced-order models," Journal of Computational and Nonlinear Dynamics 4, 034503 (2012).

790 31. M. Ghommem, I. Akhtar and M. R. Hajj, "A low-dimensional tool for predicting force decomposition coeeficients and varying inflow conditions," Progress in Computational Fluid Dynamics 13, 368-381 (2013).

32. G. R. Liu, Mesh Free Methods, moving beyond the Finite Element Method, edited. (CRC Press, 2002).

795 33. J. Barnes and P. Hut, "A hierarchical $\mathrm{O}(\mathrm{N} \log \mathrm{N})$ force-calculation algorithm," Nature 324, 446-449 (1986). 
34. L. Greengard and W. D. Gropp, "A parallel version of the fast multipole method," Comput Math Appl 20, 63-71 (1990).

35. R. W. Hockney and J. W. Eastwood, Computer simulation using particles, edited. (Institute of Physics Publishing, 1988).

36. L. Verlet, "Computer experiments on classical fluids I. Thermodynamical properties of Lennard-Jones molecules," Phys Rev 159, 98-103 (1967).

37. L. Cao, Q. H. Qin and N. Zhao, "An RBF-MFS model for analyzing thermal behavior of skin tissues," International Journal of Heat and Mass Transfer 53, 12981307 (2010).

38. H. Zhang, "Lattice Boltzmann method for solving the bioheat equation," Physics in medicine and biology 53, N15-23 (2008).

39. S. C. Jiang and X. X. Zhang, "Effects of dynamic changes of tissue properties during laser-induced interstitial thermotherapy (LITT)," Lasers in medical science 19, 197-202 (2005).

40. R. A. London, M. E. Glinsky, G. B. Zimmerman, D. S. Bailey, D. C. Eder and S. L. Jacques, "Laser-tissue interaction modeling with LATIS," Applied optics 36, 90689074 (1997). 